\title{
Brain Metastasis in Patients with Small Cell Lung Cancer
}

\section{$\mathrm{Na} \mathrm{Li}(\mathbb{D}$ \\ Yuxin Chu (D) \\ Qibin Song}

Cancer Center, Renmin Hospital of Wuhan University, Wuhan, 430060,

People's Republic of China
Correspondence: Qibin Song

Email qibinsong@whu.edu.cn
Purpose: To characterize the risk factors for brain metastasis (BM) at presentation and analyze the prognostic factors for patients with small cell lung cancer (SCLC).

Patients and Methods: Patients were recruited from the SEER database between 2010 and 2016. They were divided into two groups according to BM status. The incidence trends of SCLC and its BM were analyzed by joinpoint software. The risk factors for BM in SCLC were identified by binary logistic regression models. The prognostic factors for SCLC patients with BM were identified by Cox proportional hazard models.

Results: The incidence of SCLC and its BM significantly decreased after 2010. Totally 11,093 patients were collected, including 1717 (15.5\%) patients with BM and 9376 (84.5\%) patients without BM. In multivariate logistic regression analysis, age, male and higher $\mathrm{T}$ stage were independent risk factors for BM in SCLC patients at presentation. SCLC patients with BM showed inferior survival to those without BM. In multivariate Cox regression analysis, increasing age, large tumor size, and higher $\mathrm{N}$ stage were risk factors for poor prognosis, while other race, surgery, adjuvant radiotherapy, and chemotherapy were protective factors for SCLC patients with BM. A nomogram was developed for prognosis evaluation of such patients.

Conclusion: Age, male and higher T stage were risk factors for BM in SCLC patients at presentation. Increasing age, large tumor size, and advanced $\mathrm{N}$ stage may predict poor survival for SCLC patients with BM. Multidisciplinary therapies may provide clinical benefits. This study will help identify patients with higher BM risk and hopefully improve their clinical outcome.

Keywords: small cell lung cancer, SCLC, brain metastasis, BM, survival, risk factor

\section{Introduction}

Small cell lung cancer (SCLC) is a highly aggressive disease, which represents approximately $20 \%$ of all lung cancers. ${ }^{1}$ As a unique subtype of lung cancer, SCLC disseminates early and often manifests at extensive stages, ${ }^{2}$ constituting one of the leading cause of cancer death. It is conservatively estimated that $10-20 \%$ of SCLC patients are amalgamated with brain metastasis (BM) at onset, and $50-80 \%$ of them will eventually develop BM during the treatment process. ${ }^{3} \mathrm{BM}$ is a more aggressive health problem, with an annual incidence of $8.3-14.3$ per 100,000 people. ${ }^{4} \mathrm{BM}$ has become a serious threatening factor for the patients' survival. According to different tissue sources, BM has unique clinicopathological patterns. ${ }^{4}$ Patients with BM often have dismal prognoses, with a short median survival. ${ }^{5}$ Treatments for symptomatic $\mathrm{BM}$ are mainly based on local approaches, including surgical resection, stereotactic radiosurgery (SRS) and whole-brain radiation therapy (WBRT). ${ }^{6}$ In recent years, 
small-molecule tyrosine kinase inhibitor (TKI) afatinib has been introduced to treat patients with BM. Its clinical trials have demonstrated prolonged survival and improved quality of life. Lung cancer patients with BM can still benefit from immunotherapy. The PD-1/PD-L1 inhibitor pembrolizumab and relevant antibodies are involved in the treatment of lung cancer patients with BM. ${ }^{7}$ However, current approved treatments do not improve the survival for every patient. The high incidence and detriment of BM make it imperative to identify the risk factors for BM, so that doctors can early recognize such patients and deliver appropriate treatment in time. ${ }^{8}$ Furthermore, it is also crucial to characterize the prognostic factors for SCLC patients with BM, because better understanding of these factors may contribute to their prognosis evaluation and improve the management of this disease.

With the help of the Surveillance, Epidemiology, and End Results (SEER) database, we have extracted a large population of SCLC patients for our study. The purpose of this investigation is to analyze the clinicopathological features of SCLC patients and identify the risk factors for BM at presentation. Moreover, we also analyzed the prognostic factors for SCLC patients with BM. The information from our study may provide insights into the risk factors for development of BM prior to detection and contribute to the tailored therapy for these patients.

\section{Materials and Methods}

\section{Patient Selection}

Patients were collected from the SEER 18-registry database. This is an openly accessible database for cancer incidence and survival from 18 cancer registries, covering about $28 \%$ of the American population. ${ }^{9}$ We acquired the specific data by SEER-stat software (version 8.3.8). This study was implemented according to the Declaration of Helsinki. The inclusion criteria for selecting eligible patients were as follows: 1) the patients were diagnosed from 2010 to 2016; 2) the primary sites were lung and bronchus; 3 ) the sequence number included one primary only and first of two or more primaries; 4) the ICD-O-3 codes for SCLC were "8002/3: Malignant tumor, small cell type", "8041/3: Small cell carcinoma, NOS", "8042/3: Oat cell carcinoma", "8043/3: Small cell carcinoma, fusiform cell", "8044/3: Small cell carcinoma, intermediate cell", "8045/3: Combined small cell carcinoma". Patients with either bone or liver metastasis were excluded. Cases with unknown information in any variables were also excluded.

\section{Table Variables}

The following variables were selected for those eligible patients: age, gender, race, tumor size, T stage, $\mathrm{N}$ stage, surgery, radiotherapy, chemotherapy, survival months, and SEER cause-specific death. The continuous variables were divided into categorical variables for efficient statistical analysis, including tumor size. The optimal thresholds of bifurcation in tumor size were determined by the X-tile software. Our $\mathrm{X}$-tile program could cut the tumor size into 3 subgroups for all possible divisions. A chi-square value was calculated for every possible division. The optimal division of tumor size was identified by choosing the largest chi-square value. ${ }^{10}$ The $\mathrm{X}$-tile program selected the thresholds of tumor size at 28 and $45 \mathrm{~mm}$ in terms of cancer-specific survival (CSS) (Figure 1).

\section{Statistical Analysis}

The incidence rates of SCLC and BM were calculated per 100,000 persons and age-adjusted to the 2000 US Standard

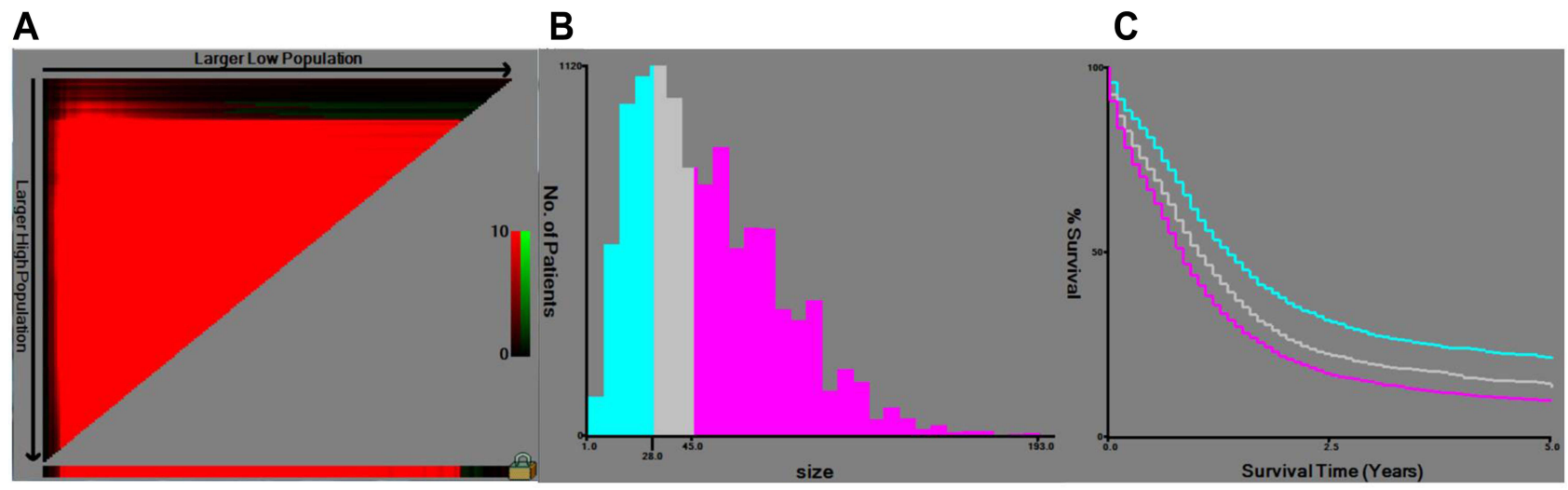

Figure I The thresholds of bifurcation in tumor size were determined by the X-tile software. (A) The "lock" symbol denotes the optimal threshold of tumor size has been found. (B) A histogram shows the cutoff values of tumor size at 28 and $45 \mathrm{~mm}$. (C) KM plots were produced according to the cutoff values. 
A

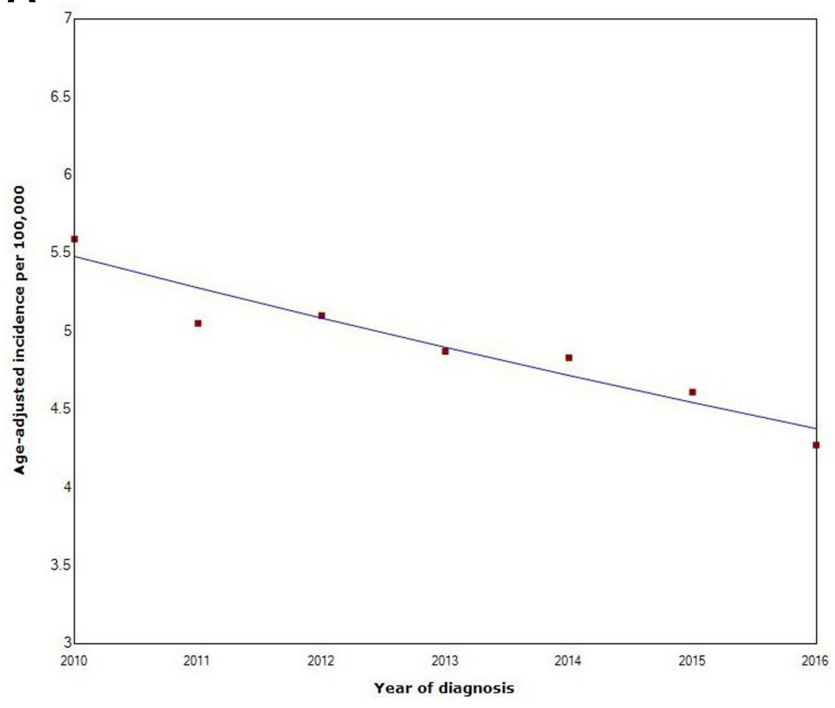

B

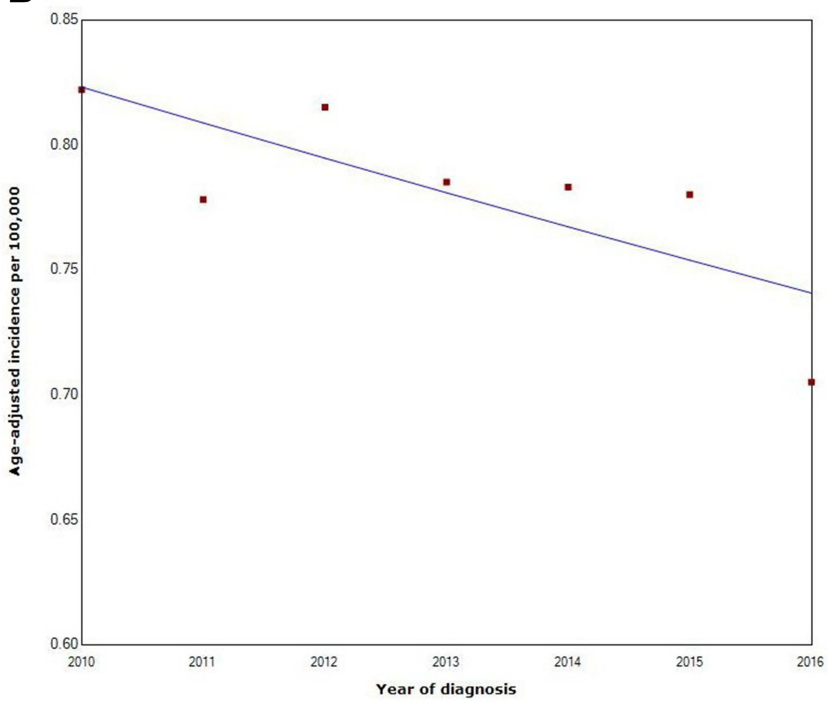

Figure 2 (A) Age-adjusted incidence rate of SCLC from 2010 to 2016. (B) Age-adjusted incidence rate of BM in SCLC from 2010 to 2016.

Population. The annual percent change (APC) from 2010 to 2016 was analyzed by joinpoint software. Patients' baseline clinical features between the non-BM and $\mathrm{BM}$ groups were listed by descriptive statistics. A student's $t$-test was used for the continuous variables age. The categorical variables were evaluated by chi-square test, which were depicted as numbers and percent frequencies. The possible risk factors relevant to BM were initially screened by univariate logistic regression analysis. The confounding factors were further adjusted by multivariate logistic regression analysis. The CSS of specific patients was estimated by Kaplan-Meier method with Log rank test. Univariate and multivariate Cox regression analyses were performed to characterize the prognostic factors of CSS for SCLC patients with BM. The independent prognostic factors from Cox regression analysis were included to construct a nomogram for predicting the 1 and 2-year CSS of such patients. All statistical analyses were completed by SPSS version 25 (SPSS Inc, Chicago, USA). The tests were 2 -sided, and $\mathrm{p}<0.05$ was statistically significant.

\section{Results}

\section{Incidence Trend Analysis}

The incidence of SCLC was steadily decreasing from 2010 to 2016, with an $\mathrm{APC}=-3.67(\mathrm{P}<0.05)$ (Figure 2A). The ageadjusted incidence rate of SCLC was 5.59/100,000 persons in 2010 but decreased to $4.27 / 100,000$ persons in 2016. In parallel, the incidence of BM in SCLC also showed a downward trend in the same period, with an $\mathrm{APC}=-1.74$ $(\mathrm{P}<0.05)$ (Figure 2B). The rate of $\mathrm{BM}$ was 0.822 /
100,000 persons in 2010 but reduced to $0.705 / 100,000$ persons in 2016. Obviously, the incidence trend of SCLC and its BM was significantly decreasing after 2010.

\section{Clinicopathological Characteristics}

According to the criteria above, a sum of 11,093 patients were recruited, including 1717(15.5\%) patients with BM and $9376(84.5 \%)$ patients without BM. In demographic features, the BM patients were significantly younger, more male $(51.95 \%$ vs $45.76 \%)$, and more black race $(12.23 \%$ vs $10.26 \%)$ than the non-BM patients $(\mathrm{p}<0.05)$. In terms of cancer characteristics, more BM patients had larger tumor size ( $>45 \mathrm{~mm}: 53.17 \%$ vs $49.45 \%)$ and $\mathrm{T} 4$ stage $(36.05 \%$ vs $33.98 \%)$ than the non-BM patients $(\mathrm{p}<0.001)$. As for treatment, the BM group had less surgery (1.63\% vs $5.79 \%)$, and more adjuvant radiotherapy $(21.03 \%$ vs $15.51 \%)$. Additionally, the distribution of $\mathrm{N}$ stage had no significant difference between the non-BM group and $\mathrm{BM}$ group $(p>0.05)$. The clinical characteristics of recruited patients are available in Table 1.

\section{Risk Factors for BM at Presentation}

We used logistic regression models to identify the risk factors for BM in SCLC patients at presentation. In univariate logistic regression analysis, the risk factors significantly associated with BM were black race, tumor size $>45 \mathrm{~mm}$, T stage $>\mathrm{T} 1(\mathrm{OR}>1, \mathrm{P}<0.05)$. In multivariate logistic regression analysis, age, sex, and $\mathrm{T}$ stage were still significantly associated with the risk of $\mathrm{BM}(\mathrm{P}<0.05)$. Consequently, age, 
Table I Characteristics of SCLC Patients Divided by Brain Metastasis $(n=\mid 1,093)$

\begin{tabular}{|c|c|c|c|c|}
\hline Variable & $\begin{array}{l}\text { No BM, } n(\%) \\
n=9376(84.5)\end{array}$ & $\begin{array}{c}\text { BM, n(\%) } \\
n=17 \mid 7(15.5)\end{array}$ & $\begin{array}{c}\text { Total, n(\%) } \\
n=11,093\end{array}$ & P-value \\
\hline Age (years) & & & & $<0.001 *$ \\
\hline Median(range) & 67(29-95) & $65(36-91)$ & $67(29-95)$ & \\
\hline Sex & & & & $<0.001$ \\
\hline Male & $4290(45.76)$ & $892(51.95)$ & $5 \mid 82(46.7 \mid)$ & \\
\hline Female & $5086(54.24)$ & $825(48.05)$ & $5911(53.29)$ & \\
\hline Race & & & & 0.030 \\
\hline White & $7952(84.81)$ & $1434(83.52)$ & $9386(84.61)$ & \\
\hline Black & $962(10.26)$ & $210(12.23)$ & $1172(10.57)$ & \\
\hline Others & $462(4.93)$ & $73(4.25)$ & $535(4.82)$ & \\
\hline Tumor size $(\mathbf{m m})$ & & & & 0.005 \\
\hline$<28$ & $2534(27.03)$ & $404(23.53)$ & $2938(26.49)$ & \\
\hline $28-45$ & $2206(23.53)$ & $400(23.3)$ & $2606(23.49)$ & \\
\hline$>45$ & $4636(49.45)$ & $913(53.17)$ & $5549(50.02)$ & \\
\hline T Stage & & & & $<0.001$ \\
\hline TI & $1676(17.88)$ & $236(13.74)$ & $1912(17.24)$ & \\
\hline $\mathrm{T} 2$ & $2519(26.87)$ & $496(28.89)$ & $3015(27.18)$ & \\
\hline T3 & $1995(21.28)$ & $366(21.32)$ & $2361(21.28)$ & \\
\hline $\mathrm{T} 4$ & $3186(33.98)$ & $619(36.05)$ & $3805(34.3)$ & \\
\hline N Stage & & & & 0.991 \\
\hline No & $1870(19.94)$ & $340(19.8)$ & $2210(19.92)$ & \\
\hline NI & $824(8.79)$ & $149(8.68)$ & $973(8.77)$ & \\
\hline N2 & $4968(52.99)$ & $909(52.94)$ & $5877(52.98)$ & \\
\hline N3 & $17 \mid 4(\mid 8.28)$ & $319(18.58)$ & $2033(18.33)$ & \\
\hline Surgery & & & & $<0.001$ \\
\hline No & $8833(94.21)$ & $1689(98.37)$ & $10,522(94.85)$ & \\
\hline Yes & $543(5.79)$ & $28(1.63)$ & $57 I(5.15)$ & \\
\hline Radiotherapy & & & & $<0.001$ \\
\hline No & $7894(84.19)$ & 1346(78.39) & $9240(83.3)$ & \\
\hline Adjuvant & $1454(|5.5|)$ & $361(21.03)$ & $1815(16.36)$ & \\
\hline Neo-adjuvant & $28(0.3)$ & $10(0.58)$ & $38(0.34)$ & \\
\hline Chemotherapy & & & & $<0.001$ \\
\hline No/unknown & $2146(22.89)$ & $497(28.95)$ & $2643(23.83)$ & \\
\hline Yes & $7230(77.11)$ & $1220(71.05)$ & $8450(76.17)$ & \\
\hline
\end{tabular}

Note: *Student's t-test was used for the continuous variables age.

Abbreviation: BM, brain metastasis.

male and advanced $\mathrm{T}$ stage were independent risk factors for BM in SCLC patients at presentation. The specific results of logistic regression analysis for BM are displayed in Table 2.

\section{Survival Analysis}

Kaplan-Meier survival analysis indicated that the CSS of patients with BM was significantly worse than those without BM (Figure 3, $\mathrm{p}<0.0001$ ). To be concrete, the median CSS for patients without $\mathrm{BM}$ was 13 months $(95 \% \mathrm{CI}=12.6-13.4)$, while the median CSS for patients with BM was only 6 months (95\% CI=5.5-6.5) during the study period (Table 3).
Consequently, BM in SCLC patients usually portends unfavorable outcome.

\section{Identify Prognostic Factors for SCLC Patients with BM}

We also used Cox regression analysis to identify the prognostic factors for those SCLC patients with BM. In univariate Cox regression model, age, tumor size $>45 \mathrm{~mm}$, T4 stage, and $\mathrm{N}$ stage $>\mathrm{N} 1$, surgery, adjuvant radiotherapy, and chemotherapy were significantly associated with the CSS of such patients $(\mathrm{P}<0.05)$. In multivariate Cox 
Table 2 Risk Factors Associated with BM in SCLC Patients $(n=\mid 1,093)$

\begin{tabular}{|c|c|c|c|c|}
\hline \multirow[t]{2}{*}{ Variable } & \multicolumn{2}{|c|}{ Univariate Logistic Model } & \multicolumn{2}{|c|}{ Multivariate Logistic Model } \\
\hline & OR(95\% Cl) & P-value & OR(95\% Cl) & P-value \\
\hline Age (years) & $0.98(0.975-0.985)$ & $<0.001$ & $0.98 I(0.976-0.986)$ & $<0.001$ \\
\hline \multicolumn{5}{|l|}{ Sex } \\
\hline Male & Reference & & Reference & \\
\hline Female & $0.78(0.704-0.865)$ & $<0.001$ & $0.785(0.707-0.87 I)$ & $<0.001$ \\
\hline \multicolumn{5}{|l|}{ Race } \\
\hline White & Reference & & Reference & \\
\hline Black & $1.211(1.03-1.417)$ & 0.019 & $1.143(0.97 I-1.34)$ & 0.072 \\
\hline Others & $0.876(0.675-1.121)$ & 0.306 & $0.882(0.678-1.13)$ & 0.27 \\
\hline \multicolumn{5}{|c|}{ Tumor size (mm) } \\
\hline$<28$ & Reference & & Reference & \\
\hline $28-45$ & $1.137(0.979-1.321)$ & 0.092 & $0.977(0.819-1.167)$ & 0.8 \\
\hline$>45$ & $1.235(1.089-1.403)$ & 0.001 & $1.017(0.865-1.199)$ & 0.839 \\
\hline \multicolumn{5}{|l|}{ T Stage } \\
\hline TI & Reference & & Reference & \\
\hline $\mathrm{T} 2$ & $1.398(1.185-1.654)$ & $<0.001$ & $1.369(1.114-1.684)$ & 0.003 \\
\hline T3 & $1.303(1.093-1.555)$ & 0.003 & $1.26(1.023-1.553)$ & 0.03 \\
\hline $\mathrm{T} 4$ & $1.38(1.176-1.624)$ & $<0.001$ & $1.31(1.067-1.609)$ & 0.01 \\
\hline \multicolumn{5}{|l|}{ N Stage } \\
\hline No & Reference & & Reference & \\
\hline NI & $0.995(0.805-1.224)$ & 0.959 & $0.982(0.794-1.21)$ & 0.869 \\
\hline N2 & $1.006(0.88-1.153)$ & 0.927 & $0.96(0.837-1.103)$ & 0.561 \\
\hline N3 & $1.024(0.867-1.209)$ & 0.783 & $0.943(0.796-1.117)$ & 0.498 \\
\hline
\end{tabular}

Abbreviations: $\mathrm{OR}$, odds ratio; $\mathrm{Cl}$, confidence interval.

regression model for adjusting confounders, increasing age, tumor size $>45 \mathrm{~mm}$, N stage $>\mathrm{N} 1$ were independent risk factors for poor survival $(\mathrm{HR}>1, \mathrm{P}<0.05)$. By contrast, other race, surgery, adjuvant radiotherapy, and chemotherapy were independent protective factors for SCLC patients with $\mathrm{BM}(\mathrm{HR}<1, \mathrm{P}<0.05)$. The concrete results of prognostic factors are listed in Table 4.

\section{Nomogram for Prognosis Evaluation}

A nomogram was constructed on the basis of independent prognostic factors to evaluate the CSS of SCLC patients with BM (Figure 4). By inputting a patient's information on these prognostic factors, the nomogram will provide a pragmatic model for the doctors to evaluate the 1-year and 2-year survival for SCLC patients with BM. In this nomogram, we can draw a vertical line from each prognostic factor to the Points scale to acquire respective points. The points of each variate can be added to produce a total point. ${ }^{11}$ Then, we can draw a vertical line from the Total Points scale to the 1-year and 2-year survival scale to estimate the probabilities of survival for each patient. ${ }^{11}$

\section{Discussion}

As an aggressive cancer, SCLC is conspicuous with a high risk of BM. According to our analysis of the SEER database, $15.5 \%$ of the SCLC patients presented with BM at presentation. The incidence of SCLC and its BM has significantly decreased after 2010. Age, male and advanced $\mathrm{T}$ stage independently portended greater odds of BM at presentation. SCLC patients with BM showed inferior survival to those without BM. Moreover, increasing age, large tumor size, and advanced $\mathrm{N}$ stage were predictors for poor survival of SCLC patients with BM. These results may have some clinical implications.

Our incidence trend analysis indicated significant downward trends for both SCLC and its BM ever since 2010. A recent study reported that the incidence of SCLC declined by $3.6 \%$ annually from 2001 to 2016 . $^{12}$ Just similarly, our results also showed an APC of -3.67 in SCLC since 2010. The remarkable incidence decrease may be interpreted by the reduction of smoking in America in recent years. ${ }^{12}$ The decrease of $\mathrm{BM}$ incidence in SCLC is our new finding; further research is necessary to elucidate the biological etiology of this trend. With 


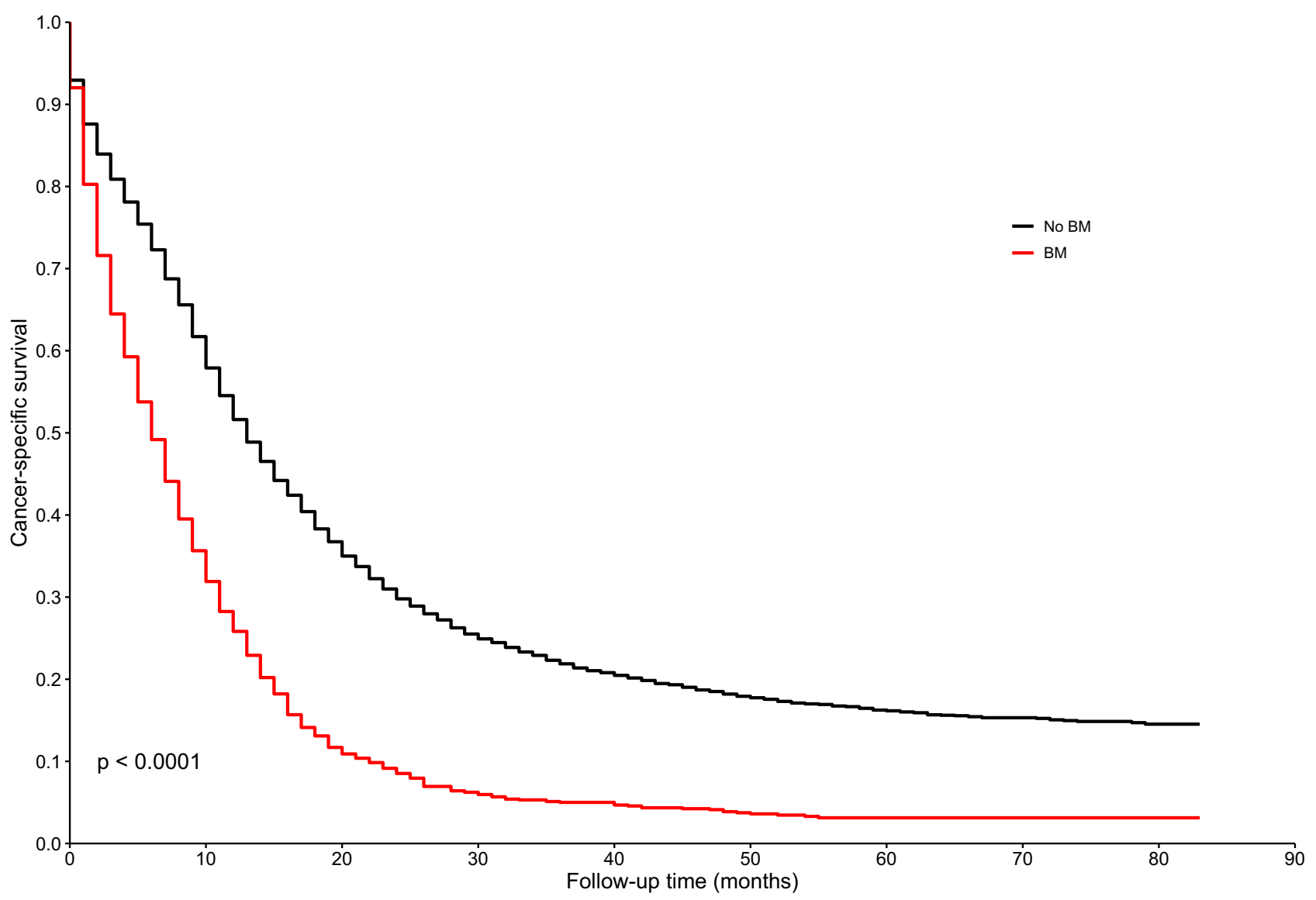

Figure 3 Kaplan-Meier curves indicating the CSS for SCLC patients based on the existence of BM.

respect to the risk factors for BM of SCLC patients at presentation, a recent study has considered that lung cancer patients were inclined to live longer owing to the progress in systemic therapy for cancer, so they had more time to develop BM. ${ }^{13}$ Consistent with this report, we have also identified age as an independent risk factor for BM in those SCLC patients. We speculate that younger patients may live longer than their older counterparts, so they still tend to have more time to yield BM. Furthermore, another study has analyzed the risk factors for $\mathrm{BM}$ in 153 patients with limited-stage SCLC. It

Table 3 Compare the Median Survival of the SCLC Patients $(n=\mid 1,093)$

\begin{tabular}{|l|c|c|}
\hline & Patients, N & $\begin{array}{c}\text { Median CSS } \\
95 \% \text { Cl, Months }\end{array}$ \\
\hline No BM & 9376 & $13(12.6-13.4)$ \\
BM & 1717 & $6(5.5-6.5)$ \\
P-value & & $<0.001$ \\
\hline
\end{tabular}

Abbreviations: BM, brain metastasis; CSS, cancer-specific survival. reported that patients with higher $\mathrm{T}$ stage had an increased risk of BM. ${ }^{14}$ Evidently, the T stage represents the status of primary site, and higher $\mathrm{T}$ stage reflects the more aggression of cancer. Our study has also indicated that SCLC with higher $\mathrm{T}$ stage was an independent risk factor for a higher odds of BM. Additionally, some variables, such as hypoxia, may cause the aggressiveness and fast progression of cancer. The hypoxia condition may upregulate the hypoxia-inducible factor (HIF); this helix transcription factor can affect the carcinogenesis and tumor growth by regulating the genes in glycolytic metabolism, angiogenesis, and other biological process. ${ }^{15}$ Hence, age, advanced T stage, and hypoxia may become the predictors for BM in patients with SCLC.

As far as the prognostic factors for SCLC patients with BM are concerned, a recent study has revealed that age $>60$ was a reliable marker for poor clinical outcome. It is suggested that screening for BM should be applied for these patients. ${ }^{16}$ Our study also identified that increasing age portended a poor prognosis. Older patients may have dismal performance status and more treatment-associated toxicity. Moreover, another 
Table 4 The Prognostic Factors Relevant to the CSS of SCLC Patients with BM $(n=1717)$

\begin{tabular}{|c|c|c|c|c|}
\hline \multirow[t]{2}{*}{ Characteristics } & \multicolumn{2}{|l|}{ Univariate Cox } & \multicolumn{2}{|l|}{ Multivariate Cox } \\
\hline & HR(95\% Cl) & P-value & HR(95\% Cl) & P-value \\
\hline Age (years) & $1.025(1.02-1.031)$ & $<0.001$ & $1.016(1.01-1.022)$ & $<0.001$ \\
\hline \multicolumn{5}{|l|}{ Sex } \\
\hline Male & Reference & & Reference & \\
\hline Female & $0.948(0.858-1.048)$ & 0.296 & $0.958(0.866-1.06)$ & 0.403 \\
\hline \multicolumn{5}{|l|}{ Race } \\
\hline White & Reference & & Reference & \\
\hline Black & $0.982(0.842-I .145)$ & 0.815 & $0.873(0.747-1.02)$ & 0.087 \\
\hline Others & $0.802(0.624-\mid .03 I)$ & 0.085 & $0.6 \mathrm{II}(0.474-0.789)$ & $<0.001$ \\
\hline \multicolumn{5}{|c|}{ Tumor size (mm) } \\
\hline$<28$ & Reference & & Reference & \\
\hline $28-45$ & $1.135(0.981-1.315)$ & 0.09 & I.I6(0.967-I.392) & 0.11 \\
\hline$>45$ & $1.225(I .082-1.387)$ & 0.001 & $1.292(1.095-1.523)$ & 0.002 \\
\hline \multicolumn{5}{|l|}{ T Stage } \\
\hline TI & Reference & & Reference & \\
\hline $\mathrm{T} 2$ & $1.054(0.895-I .24 I)$ & 0.532 & $0.989(0.8-1.223)$ & 0.92 \\
\hline $\mathrm{T} 3$ & $1.148(0.966-1.363)$ & 0.117 & $1.01(0.82-1.245)$ & 0.923 \\
\hline $\mathrm{T} 4$ & $1.185(1.012-1.388)$ & 0.035 & I.0I (0.822-I.24I) & 0.923 \\
\hline \multicolumn{5}{|l|}{ N Stage } \\
\hline No & Reference & & Reference & \\
\hline $\mathrm{NI}$ & $0.99(0.806-1.218)$ & 0.927 & $1.087(0.883-1.338)$ & 0.431 \\
\hline N2 & I.306(I.I42-I.493) & $<0.001$ & $1.56(1.36-1.788)$ & $<0.001$ \\
\hline N3 & $1.275(1.083-1.501)$ & 0.004 & $1.504(1.273-1.777)$ & $<0.001$ \\
\hline \multicolumn{5}{|l|}{ Surgery } \\
\hline No & Reference & & Reference & \\
\hline Yes & $0.442(0.28 I-0.695)$ & $<0.001$ & $0.596(0.375-0.945)$ & 0.028 \\
\hline \multicolumn{5}{|l|}{ Radiotherapy } \\
\hline No & Reference & & Reference & \\
\hline Adjuvant & $0.7 \mid 8(0.634-0.812)$ & $<0.001$ & $0.848(0.746-0.963)$ & 0.011 \\
\hline Neo-adjuvant & I.105(0.593-2.06) & 0.752 & I.I83(0.632-2.217) & 0.599 \\
\hline \multicolumn{5}{|l|}{ Chemotherapy } \\
\hline No & Reference & & Reference & \\
\hline Yes & $0.27 I(0.242-0.305)$ & $<0.001$ & $0.267(0.236-0.302)$ & $<0.001$ \\
\hline
\end{tabular}

Abbreviations: $\mathrm{HR}$, hazard ratio; $\mathrm{Cl}$ : confidence interval.

study indicated that SCLC patients with large primary tumor had significantly worse survival than the referent patients. ${ }^{17}$ In parallel, our study has likewise identified tumor size $>45 \mathrm{~mm}$ as an independent risk factor for poor survival in SCLC patients with BM. These findings reflect that large tumor size remarkably predicted unfavorable prognosis for such patients. The lymph node (LN) status is also an important prognostic factor for the lung cancer patients. A recent study has reported that LN metastasis was associated with greater odds of multiple metastases and worse prognosis. ${ }^{18}$ Our study indicated that patients at N2 and N3 stages had worse survival than those at N0 stage. It is possible that lymphatics may serve as a pathway for the cancer cells to disseminate and exacerbate the patients' survival. These findings may provide evidence to confirm the prognostic value of LN status in SCLC. Anyway, precise prognostic evaluation is the essential foundation for benefitrisk-adapted therapy for SCLC patients with BM, ${ }^{19}$ our study has analyzed the prognostic factors in a large cohort of such patients. We have also constructed a nomogram for prognosis evaluation of SCLC patients with BM. This prognostic model may optimize the current treatment strategy and survival prediction for such patients.

With respect to the therapeutic schedules in SCLC patients with BM, a multidisciplinary approach is necessary to optimize the prognosis and quality of life in lung cancer patients. ${ }^{20}$ In addition to surgery, radiotherapy 


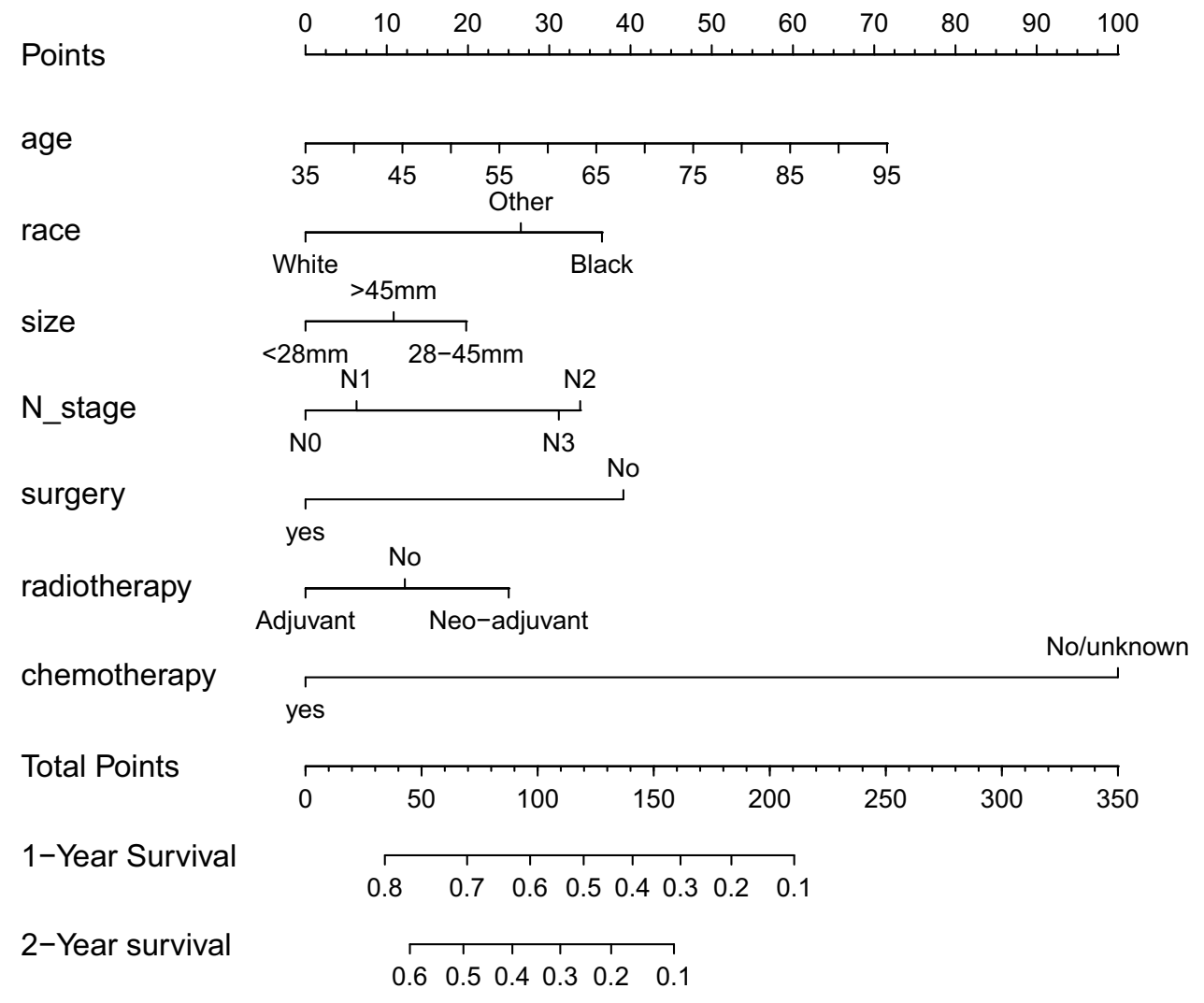

Figure 4 Nomogram for predicting the 1-year and 2-year survival of SCLC patients with BM.

plays an important role in cancer control. Prophylactic cranial irradiation (PCI) has become a widely available technique for treating both limited and extensive lung cancer. ${ }^{21}$ PCI can not only reduce the BM incidence but also improve the patients' survival, so current guidelines often recommend PCI for the lung cancer patients who have a good initial response. ${ }^{22}$ In consonance with these publications, our study also identified surgery, adjuvant radiotherapy, and chemotherapy as independent protective factors for SCLC patients with BM. These findings imply that sequential therapies with a multidisciplinary approach may well provide clinical benefit.

Several limitations should be concerned in our study. First, this is a retrospective study from the SEER database, some inherent bias cannot be evaded completely. Second, the SEER database lacks additional data regarding smoking exposure pack-years, comorbidities and previous or current possible brain vascular diseases, which may conceal some important risk factors. ${ }^{23}$ Third, as the time, sequence and combination of different treatments varied widely, ${ }^{24}$ we could not thoroughly evaluate the effect of such clinical factors on SCLC patients with BM. So, our results should be explained with caution, and future prospective clinical trials are necessary to validate our findings. So far, this is one of the largest real-life studies specifically addressing the risk factors for BM in SCLC. Therefore, this study may provide a unique opportunity to acquire deeper insight into the risk factors for BM in SCLC patients.

\section{Conclusion}

This study has found that age, male and advanced $\mathrm{T}$ stage were independent risk factors for BM in SCLC patients at presentation. Increasing age, large tumor size, and advanced $\mathrm{N}$ stage may presage poor survival for SCLC patients with BM. Multidisciplinary therapies may provide clinical benefit. A deep understanding of the risk factors for BM in SCLC patients may help us to enrich the subset of patients who are more susceptible to develop BM, more individually treat such patients, and improve their clinical outcome.

\section{Ethical Statement}

The SEER data contain no identifiers and are publicly available; ethical approval was obtained from the Institutional Review Board (IRB) of Ethics Committee in Renmin Hospital of Wuhan University. 


\section{Acknowledgments}

The authors acknowledge the SEER database for providing high-quality clinical data for our study.

\section{Funding}

This study was supported by the National Natural Science Foundation of China (81670123, 81802980).

\section{Disclosure}

All authors declare no conflicts of interest in this work.

\section{References}

1. Couñago F, de la Pinta $\mathrm{C}$, Gonzalo $\mathrm{S}$, et al. GOECP/SEOR radiotherapy guidelines for small-cell lung cancer. World J Clin Oncol. 2021;12(3):115-143. doi:10.5306/wjco.v12.i3.115

2. Noronha V, Sekhar A, Patil VM, et al. Systemic therapy for limited stage small cell lung carcinoma. $J$ Thorac Dis. 2020;12 (10):6275-6290. doi:10.21037/jtd-2019-sclc-11

3. Hou R, Li H, Cao J, Song X, Zhang X, Wang W. Validation of a novel prognostic index: BMS-score for patients with brain metastases of small cell lung cancer. Ann Palliat Med. 2021;10(1):29-36. doi:10.21037/apm-20-1819

4. Ni W, Chen W, Lu Y. Emerging findings into molecular mechanism of brain metastasis. Cancer Med-Us. 2018;7(8):3820-3833. doi:10.1002/cam4.1667

5. Liu Y, Liu XH, Wang Y, et al. A study on different therapies and prognosis-related factors for 101 patients with SCLC and brain metastases. Cancer Biol Ther. 2017;18(9):670-675. doi:10.1080/ 15384047.2017.1360450

6. Ettinger DS, Wood DE, Aisner DL, et al. NCCN guidelines insights: non-small cell lung cancer, version 2.2021. J Natl Compr Canc Netw. 2021;19(3):254-266. doi:10.6004/jncen.2021.0013

7. Rybarczyk-Kasiuchnicz A, Ramlau R, Stencel K. Treatment of brain metastases of non-small cell lung carcinoma. Int J Mol Sci. 2021;22 (2):593. doi:10.3390/ijms22020593

8. Rusthoven CG, Yamamoto M, Bernhardt D, et al. Evaluation of first-line radiosurgery vs whole-brain radiotherapy for small cell lung cancer brain metastases: the FIRE-SCLC cohort study. JAMA Oncol. 2020;6(7):1028-1037. doi:10.1001/jamaoncol.2020.1271

9. Shi T, Song X, Liu Q, et al. Survival benefit of palliative gastrectomy followed by chemotherapy in stage IV gastric signet ring cell carcinoma patients: a large population-based study. Cancer Med. 2019;8 (13):6010-6020. doi:10.1002/cam4.2521

10. Song K, Shi X, Wang H, et al. Can a nomogram help to predict the overall and cancer-specific survival of patients with chondrosarcoma? Clin Orthop Relat Res. 2018;476(5):987-996. doi:10.1007/ s11999.0000000000000152

International Journal of General Medicine

\section{Publish your work in this journal}

The International Journal of General Medicine is an international, peer-reviewed open-access journal that focuses on general and internal medicine, pathogenesis, epidemiology, diagnosis, monitoring and treatment protocols. The journal is characterized by the rapid reporting of reviews, original research and clinical studies
11. Song K, Song J, Shi X, et al. Development and validation of nomograms predicting overall and cancer-specific survival of spinal chondrosarcoma patients. Spine. 2018;43(21):E1281-E1289. doi:10.1097/ BRS.0000000000002688

12. Howlader N, Forjaz G, Mooradian MJ, et al. The effect of advances in lung-cancer treatment on population mortality. $N$ Engl J Med. 2020;383(7):640-649. doi:10.1056/NEJMoa1916623

13. Waqar SN, Waqar SH, Trinkaus $\mathrm{K}$, et al. Brain metastases at presentation in patients with non-small cell lung cancer. Am J Clin Oncol. 2018;41(1):36-40. doi:10.1097/COC.0000000000000230

14. Zheng Y, Wang L, Zhao W, et al. Risk factors for brain metastasis in patients with small cell lung cancer without prophylactic cranial irradiation. Strahlentherapie Und Onkologie: Organ der Deutschen Rontgengesellschaft [Et Al]. 2018;194(12):1152-1162. doi:10.1007/ s00066-018-1362-7

15. Pezzuto A, Carico E. Role of HIF-1 in cancer progression: novel insights. a review. Curr Mol Med. 2018;18(6):343-351. doi:10.2174/ 1566524018666181109121849

16. Krug S, Teupe F, Michl P, Gress TM, Rinke A. Brain metastases in patients with neuroendocrine neoplasms: risk factors and outcome. BMC Cancer. 2019;19(1):362. doi:10.1186/s12885-019-5559-7

17. Fukui T, Itabashi M, Ishihara $M$, et al. Prognostic factors affecting the risk of thoracic progression in extensive-stage small cell lung cancer. BMC Cancer. 2016;16:197. doi:10.1186/s12885-016-2222-4

18. Yang J, Peng A, Wang B, et al. The prognostic impact of lymph node metastasis in patients with non-small cell lung cancer and distant organ metastasis. Clin Exp Metastasis. 2019;36(5):457-466. doi:10.1007/s10585-019-09985-y

19. Steindl A, Schlieter F, Klikovits T, et al. Prognostic assessment in patients with newly diagnosed small cell lung cancer brain metastases: results from a real-life cohort. $J$ Neurooncol. 2019;145 (1):85-95. doi:10.1007/s11060-019-03269-x

20. Pezzuto A, Terzo F, Graziani ML, Ricci A, Bruno P, Mariotta S. Lung cancer requires multidisciplinary treatment to improve patient survival: a case report. Oncol Lett. 2017;14(3):3035-3038. doi:10.3892/ ol.2017.6511

21. Edelman MJ. Prophylactic cranial irradiation for small-cell lung cancer: time for a reassessment. Am Soc Clin Oncol Educ Book. 2020;40:24-28. doi:10.1200/EDBK_281041

22. Zeng H, Xie P, Meng X, et al. Risk factors for brain metastases after prophylactic cranial irradiation in small cell lung cancer. Sci Rep. 2017;7:42743. doi:10.1038/srep42743

23. Du X, Tian D, Liu L, et al. Surgery in patients with small cell lung cancer: a period propensity score matching analysis of the Seer database, 2010-2015. Oncol Lett. 2019;18(5):4865-4881. doi:10.3892/ol.2019.10792

24. Sperduto PW, Yang TJ, Beal K, et al. Estimating survival in patients with lung cancer and brain metastases: an update of the graded prognostic assessment for lung cancer using molecular markers (lung-molGPA). JAMA Oncol. 2017;3(6):827-831. doi:10.1001/ jamaoncol.2016.3834 across all disease areas. The manuscript management system is completely online and includes a very quick and fair peer-review system, which is all easy to use. Visit http://www.dovepress.com/ testimonials.php to read real quotes from published authors. 